Artículos científicos

\title{
Gestión del conocimiento como determinante de la capacidad de innovación en instituciones de educación superior
}

Knowledge management as a determinant of innovation capacity in higher education institutions

A gestão do conhecimento como determinante da capacidade de inovação em instituições de ensino superior

José Félix García Rodríguez

Universidad Juárez Autónoma de Tabasco, División Académica de Ciencias Económico Administrativas, México

jfgr55@hotmail.com https://orcid.org/0000-0002-7319-1472

Georgina Guadalupe Shriner Sierra

Universidad Juárez Autónoma de Tabasco, División Académica de Educación y Artes,

México

ginashriners@hotmail.com https://orcid.org/0000-0002-1857-7989

David Martínez Luis

Universidad Autónoma del Carmen, Facultad de Ciencias Económicas Administrativas, México dmartinez@pampano.unacar.mx https://orcid.org/0000-0002-4747-9368 


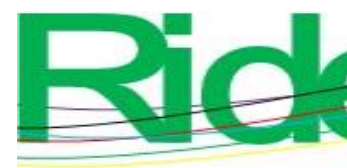

Revista Iberoamericana para la Investigación y el Desarrollo Educativo ISSN $2007-7467$

presente en las instituciones de educación superior. Resultados. Se encontró que entre las variables analizadas existe un coeficiente de correlación de 0.921 ; una relación estimada de 1.267 y estandarizada de .96 , y un valor p (0.000), lo que proporciona evidencias de la presencia de una relación positiva entre ambos constructos. Conclusiones. La gestión del conocimiento influye positivamente en la capacidad de innovación en las instituciones educativas de nivel superior estudiadas, con lo cual se confirma la hipótesis planteada.

Palabras clave: capacidad de innovación, conocimiento, gestión del conocimiento, innovación.

\section{Abstract}

Introduction. Knowledge represents the most important intangible asset of organizations to drive innovation and competitive advantage. Knowledge management translates into greater efficiency in the use of resources, better productive performance and optimal innovative processes. Objective. The purpose of the research is to analyze the relationship observed between knowledge management and the capacity for innovation in higher education institutions. The central hypothesis is that knowledge management is positively related to innovation capacity. Method. A descriptive, explanatory and confirmatory quantitative investigation was developed. Once the research problem was established and the knowledge generation and innovation variables were theoretically based, we proceeded to analyze the relationship between both constructs. Descriptive, exploratory factorial and confirmatory statistical analysis tools were used. Structural equation analysis was applied for modeling and hypothesis testing. The universe of study is 77 higher education institutions in the southeast of Mexico, where 8,603 people work. The sample size is 441 workers, and it was determined by probabilistic cluster sampling, assuming a sampling error of $5 \%$, confidence level of $95 \%$ and variance of $\mathrm{p}=.50, \mathrm{q}=.50$. The field information was obtained by applying a structured questionnaire of 88 items on the Likert scale, which was designed based on the research objectives and other instruments used in similar studies, identified during the literature review. The applied questionnaire consists of three sections. In the first, information is requested on the sociodemographic profile of the interviewees. In the second, the scale for measuring the structural capacity for knowledge management is established, based on the constructs of structure, culture, human resources and technology. Finally, the third section is 


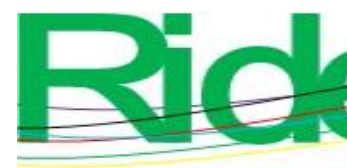
Revista Iberoamericana para la
Investigación y el Desarrollo Educativo
ISSN $2007-7467$

aimed at identifying the innovation capacity present in higher education institutions. Results. It was found that among the analyzed variables there is a correlation coefficient of 0.921 ; an estimated relationship of $(1,267)$ and a standardized relationship of $(.96)$, and a $p$ value (0.000), which provides evidence of the presence of a positive relationship between both constructs. Conclusions. Knowledge management positively influences the capacity for innovation in the higher-level educational institutions studied, thus confirming the hypothesis raised.

Keywords: Knowledge, Innovation, Knowledge Management, Innovation Capacity.

\section{Resumo}

Introdução. O conhecimento representa o ativo intangível mais importante das organizações para impulsionar a inovação e a vantagem competitiva. A gestão do conhecimento se traduz em maior eficiência no uso de recursos, melhor desempenho produtivo e processos inovadores ideais. Objetivo. O objetivo da pesquisa é analisar a relação observada entre a gestão do conhecimento e a capacidade de inovação nas instituições de ensino superior. A hipótese central é que a gestão do conhecimento está positivamente relacionada à capacidade de inovação. Método. Foi desenvolvida uma investigação quantitativa descritiva, explicativa e confirmatória. Estabelecido o problema de pesquisa e fundamentadas teoricamente as variáveis de geração de conhecimento e inovação, procedeu-se à análise da relação entre os dois construtos. Foram utilizadas ferramentas de análise estatística descritiva, exploratória e fatorial e confirmatória. A análise de equações estruturais foi aplicada para modelagem e teste de hipóteses. O universo de estudo foram 77 instituições de ensino superior no sudeste do México, onde trabalhavam 8.603 pessoas. O tamanho da amostra foi de 441 trabalhadores, e foi determinado por amostragem probabilística por conglomerados, assumindo um erro amostral de 5\%, um nível de confiança de $95 \%$ e variância de $p=0,50, q=0,50$. As informações de campo foram obtidas por meio da aplicação de um questionário estruturado de 88 itens na escala Likert, que foi elaborado com base nos objetivos da pesquisa e outros instrumentos utilizados em estudos semelhantes, identificados durante a revisão da literatura. O questionário aplicado consiste em três seções. Na primeira, são oferecidas informações sobre o perfil sociodemográfico dos entrevistados. Na segunda, estabelece-se a escala de mensuração da capacidade estrutural de gestão do conhecimento, a partir dos construtos 


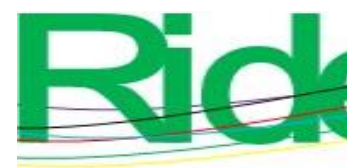
Revista Iberoamericana para la
Investigación y el Desarrollo Educativo
ISSN $2007-7467$

estrutura, cultura, recursos humanos e tecnologia. Por fim, a terceira seção visa identificar a capacidade de inovação presente nas instituições de ensino superior. Resultados. Constatouse que entre as variáveis analisadas existe um coeficiente de correlação de 0,921 ; uma relação estimada de 1.267 e uma relação padronizada de 0,96 e um valor de p (0,000), o que fornece evidências da presença de uma relação positiva entre os dois construtos. Conclusões. A gestão do conhecimento influencia positivamente a capacidade de inovação das instituições de ensino superior estudadas, confirmando a hipótese levantada.

Palavras-chave: capacidade de inovação, conhecimento, gestão do conhecimento, inovação.

Fecha Recepción: Junio 2020

Fecha Aceptación: Noviembre 2020

\section{Introducción}

En el contexto actual de globalización y economía neoliberal — con un mundo más abierto, interconectado y competitivo-, el conocimiento se ha convertido en factor clave para el desarrollo económico y social de los países y sus habitantes. El uso intensivo de la tecnología de la información exige a las economías nacionales modernizarse y prepararse para poder competir en una economía mundial donde la generación, uso y difusión del conocimiento derivado de la ciencia, la tecnología y la innovación son determinantes del éxito económico y social. Por ello, los gobiernos, las empresas, las organizaciones públicas y privadas, así como las instituciones educativas procuran mantenerse al día en conocimientos estratégicos para generar ventajas competitivas. En este contexto, la gestión de activos intangibles, como lo es el conocimiento, revisten importancia estratégica para el desarrollo y crecimiento de los países y las organizaciones (Salete, Tcholakian y Selig, 2013).

De Long y Fahey (2000) clasifican el conocimiento en tres tipos: a) conocimiento humano (lo que los individuos saben, su habilidad o pericia para hacer e incluye conocimiento tácito y explícito); b) conocimiento social (conocimiento colectivo que es más que la suma del conocimiento individual de los miembros del equipo y es en gran parte tácito, resultado de la labor conjunta), y c) conocimiento estructurado (está incorporado en los sistemas, procesos y herramientas de una organización). Al respecto, es importante mencionar que el conocimiento estructurado es explícito, ya que existe independientemente del conocimiento humano, por lo que se le considera fundamental para toda institución. Por 


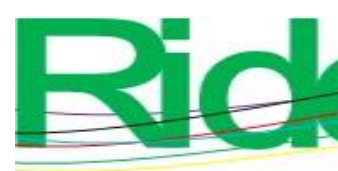

Revista Iberoamericana para la Investigación y el Desarrollo Educativo ISSN $2007-7467$

esta razón, su gestión y transferencia constituye un principio básico en la administración de las organizaciones Grant (1996).

Por gestión del conocimiento (GC) puede entenderse la habilidad individual o colectiva para generar, difundir, compartir y utilizar el conocimiento, tanto tácito como explícito. Por ello, la GC se ha convertido en una herramienta de aprendizaje útil, puesto que aporta valor dentro de una organización, economía o sociedad (Barragán, 2009). De esta manera, los procesos de innovación y ventaja competitiva de la organización resultan ser más eficientes bajo la influencia positiva de las prácticas de GC (Segarra, 2006). Por lo anterior, la GC se constituye en actividad estratégica fundamental para el desarrollo y crecimiento de toda organización, sobre todo en ambientes altamente competitivos (Drucker, 1993; Lee, Leong, Hew y Ooik, 2013; Piri, Jasemi y Abdi, 2013). Incluso, frente al capital físico y financiero, el conocimiento tiende a convertirse en uno de los activos intangibles más importantes (Reza y Pahlavani, 2013). Así, dada su importancia estratégica para la innovación y ventaja competitiva, la GC se constituye en tarea permanente de la organización (Darroch, 2005; Lee et al., 2013).

La GC surge como una filosofía estratégica para ayudar a las organizaciones a desarrollar sus capacidades para hacer frente al dinamismo y la incertidumbre del complejo entorno actual. Por medio de la adquisición sistemática, creación, intercambio y utilización de conocimientos, las organizaciones aprovechan de mejor manera sus activos y son más proactivas y adaptables a los cambios externos, desarrollando así ventajas innovadoras y competitivas (Nguyen, 2010). Para que la organización pueda generar procesos de innovación a partir de los conocimientos que posee, es necesario que implemente procesos de gestión. Los diversos enfoques de GC están enfocados en facilitar el proceso de innovación (Swan y Newell, 2000), la cual resulta ser más eficiente cuando sus trabajadores son provistos de una formación adecuada, así como de oportunidades para generar nuevas ideas (Bidmeshgipour, Khairuzzaman y Omar, 2012). Así, diversos estudios han investigado la relación existente entre la innovación y el capital humano, entendido como el conjunto de conocimientos, destrezas y habilidades que poseen los trabajadores de la organización (Bornay, De la Rosa, López y Valle, 2012). Al respecto, existen evidencias suficientes de un efecto directo y positivo de la calidad del capital humano en la innovación (Cabello, López y Valle, 2011). 


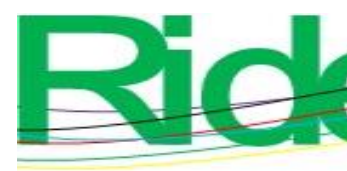

Revista Iberoamericana para la Investigación y el Desarrollo Educativo ISSN $2007-7467$

En síntesis, se puede considerar que la capacidad que tiene una organización para innovar está estrechamente relacionada con los activos intelectuales y conocimientos que posee, y que las organizaciones que gestionan los conocimientos utilizan de manera más eficiente los recursos con que cuentan, son más innovadoras y tienen un mejor desempeño (Darroch, 2005).

\section{Gestión del conocimiento}

Como se ha mencionado, el conocimiento constituye el activo más importante de la organización para la innovación y ventaja competitiva (Nonaka y Takeuchi, 1995). Por ello, las organizaciones se esfuerzan en lograr una eficiente GC par impulsar su capacidad de innovación (CIN) (Davenport y Prusak, 1998). La relación de la GC y la CIN al interior de las organizaciones se ha estudiado ampliamente. López y Meroño (2011) encontraron que la estrategia de GC tiene un impacto positivo sobre la innovación y el rendimiento productivo. El-Kot y Gamal (2011) confirmaron que existe una relación positiva y significativa entre la GC y la innovación organizacional, así como la ventaja competitiva sostenible. Por su parte, Mehrabani y Shajari (2012) observaron que la creación, organización, difusión y aplicación del conocimiento como actividades sustantivas de la GC se asocia directamente con la CIN. De la misma manera, Palacios, Gil y Garrigos (2009) hallaron que la introducción de un programa de GC en la organización coadyuva al desarrollo de habilidades de adquisición, transferencia, difusión y aplicación del conocimiento acumulado. En síntesis, estas evidencias empíricas confirman que la GC contribuye a que las organizaciones apliquen de forma efectiva sus recursos productivos, así como la CIN organizacional.

La literatura sobre GC de las organizaciones en general la sustenta en dos dimensiones: capacidad de infraestructura y capacidad de proceso. La primera corresponde a las actividades generales de la organización y comprende cuatro aspectos: estructura organizacional, cultura organizacional, recursos humanos y tecnología de la información. La segunda corresponde a la coordinación estructurada creada con el fin de gestionar eficazmente el conocimiento y resulta esencial, ya que permite a la organización capturar, procesar y transferir el conocimiento, así como gestionar eficazmente el conocimiento interno y externo (Gold, Malhotra y Segars, 2001). Está integrada por la adquisición, conversión, aplicación y protección del conocimiento (Gold et al., 2001; Lee y Choi, 2003; Nguyen, 2010). 


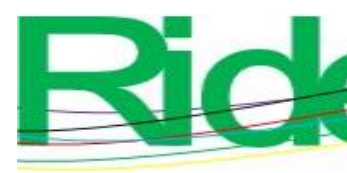

Revista Iberoamericana para la Investigación y el Desarrollo Educativo ISSN 2007 - 7467

Asimismo, se destaca la importancia de las capacidades estructurales de la organización (cultura, estructura funcional, recursos humanos y tecnológicos) como aspectos estratégicos que impulsan los procesos de adquisición, retención, transferencia y utilización del conocimiento (Chuang, 2004; Lee y Choi, 2003). Por ello, en esta investigación se considera que las capacidades estructurales de la organización constituyen aspectos estratégicos de la GC, ya que determinan la relación de la capacidad de GC con la capacidad de innovación. En síntesis, la GC debe entenderse como un mecanismo institucional que hace posible que el conocimiento sea creado, explotado y compartido (Palacios et al., 2009), y este proceso de intercambio de conocimientos conlleva a la generación de nuevas ideas, procesos y productos, es decir, a la innovación (Camelo, García, Sousa y Valle, 2011).

\section{Innovación}

El constructo innovación tiene implícita la adopción de una nueva idea o comportamiento de una organización (Damanpour, Walker y Avellaneda, 2009). A nivel organizacional, la innovación se entiende como la adopción por primera vez de una tecnología, estrategia o práctica de gestión, o una significativa reestructuración o mejora de un proceso (Haiyang y Kwaku, 2001).

La innovación puede adoptar muchas formas. En función de los resultados que produce, puede ser de proceso, producto y servicio (Prajogo y Pervaiz, 2006). De acuerdo con el nivel de alteración o cambio que introduce, puede ser incremental y radical (Darroch, 2005). Las innovaciones tecnológicas corresponden a las modificaciones incorporadas en los productos y procesos actuales basadas en la aplicación de tecnologías (Lee et al., 2013). La innovación no técnica u organizativa involucra a la estructura funcional y los procesos administrativos y de gestión (Abdullah y Hassan, 2013). La innovación técnica se divide en innovación de productos (nuevos productos o servicios introducidos para cumplir con un usuario externo o necesidad del mercado) e innovación de proceso (se refiere a los nuevos elementos introducidos en las operaciones de producción o servicios de una organización) que puede mejorar las operaciones, ahorrar costos, aumentar la eficiencia, la productividad y el rendimiento en un corto tiempo (Shu, Page, Gao y Jiang, 2012).

Al respecto, Damanpour et al. (2009) distinguen entre dos tipos de innovación de producto (de bienes y de servicios), y dos tipos de innovación de proceso: innovaciones en los procesos operativos (como los servicios al cliente, logística y adquisiciones), y las 


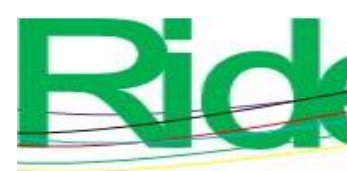

Revista Iberoamericana para la Investigación y el Desarrollo Educativo ISSN 2007-7467

innovaciones en los procesos de gestión (como la planificación estratégica, el proyecto de gestión y evaluación de los empleados). Asimismo, clasifican tres tipos de innovación que son aplicables a las organizaciones de servicios: de servicio, de procesos tecnológicos y de procesos administrativos. Respecto a la innovaciones de servicio, Damanpour et al. (2009) mencionan que en la investigación de la innovación no se ha distinguido por lo general entre las innovaciones de producto y las de servicio, lo cual se debe a que ambas tienen un enfoque externo, son impulsadas principalmente por los mercados, y sus resultados son la introducción de cambios en la producción de la organización para sus consumidores o clientes. Al igual que las innovaciones de productos, los motores de la innovación de servicios son la demanda y el deseo de introducir nuevos servicios para los mercados existentes o nuevos nichos de mercado.

En lo que corresponde a innovaciones de proceso, estos mismos autores dicen que contrario a las innovaciones de productos o servicios, las de proceso tienen un enfoque interno, ya que tienen por objeto aumentar la eficiencia y la eficacia de la organización para facilitar la producción y la entrega de bienes o servicios a los clientes. Los nuevos procesos pueden estar asociados con el núcleo tecnológico o sistema técnico de la organización (innovaciones de procesos tecnológicos), o con el núcleo administrativo de la organización (innovaciones de proceso administrativo). Las innovaciones de procesos tecnológicos constituyen nuevos elementos introducidos en el sistema productivo y de servicios de la organización. Este tipo de innovación busca reducir los tiempos de entrega, aumentar la flexibilidad operativa y reducir los costos de producción. Por lo tanto, las innovaciones de procesos tecnológicos modifican los procesos y sistemas operativos de la organización. En las organizaciones de servicios, estas innovaciones están asociadas a la tecnología de la información, por lo que se conoce también como innovación tecnológica.

Finalmente, las innovaciones en los procesos administrativos corresponden a nuevos enfoques y prácticas para motivar y recompensar a los miembros de la organización, diseñar la estrategia y la estructura de las tareas y unidades, y modificar los procesos de gestión de la organización. Las innovaciones tecnológicas están directamente relacionadas con el trabajo y actividad principal de la organización para producir cambios en sus sistemas operativos, y las innovaciones administrativas están indirectamente relacionadas con la actividad básica de trabajo de la organización y afectan principalmente a sus sistemas de gestión. Las innovaciones administrativas de proceso se refieren a los cambios en la 


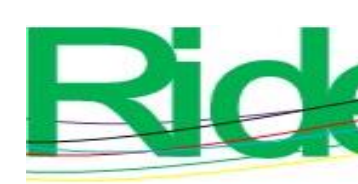

Revista Iberoamericana para la Investigación y el Desarrollo Educativo ISSN $2007-7467$

estructura y los procesos de la organización, sistemas administrativos, conocimientos utilizados en la realización de los trabajos de gestión, y capacidad de gestión que le permiten funcionar y tener éxito mediante el uso de sus recursos con eficacia. Este tipo de innovación se conoce también como innovación administrativa (Damanpour et al., 2009). En el contexto actual de modernidad y uso intensivo de las tecnologías de comunicación, para las instituciones de educación superior resulta fundamental el desarrollo y aplicación de innovaciones tecnológicas en sus procesos administrativos y académicos.

Queda claro entonces que las organizaciones capaces de gestionar el conocimiento (CGC) obtienen mejores resultados, y que este es el motor principal de innovación y ventaja competitiva (Nonaka y Takeuchi, 1995). Sin embargo, para que el conocimiento sea creado y explotado tiene que ser compartido, lo que a su vez sirve para generar nuevas ideas, procesos y productos, es decir, innovación (Camelo et al., 2011). Por ello, la CGC debe asumirse como tarea permanente para impulsar la CIN institucional (Palacios et al., 2009). Las investigaciones de Nonaka y Takeuchi (1995) sobre la creación de conocimiento organizativo conciben el conocimiento como requisito principal para la innovación y ventaja competitiva. En esta línea de investigación, el propósito central de esta investigación es analizar la relación existente entre la CGC y la CIN en instituciones de educación superior.

En el análisis, la capacidad de innovación se asume como variable dependiente y está integrada por la innovación de productos y la innovación de procesos. La primera se refiere al desarrollo o mejora de nuevos productos y servicios introducidos a mercados ya existentes o nuevos (Wang y Ahmed, 2004; Damanpour et al., 2009). La segunda corresponde a nuevos métodos de producción y/o enfoques de gestión (Damanpour et al., 2009; Wang y Ahmed, 2004). Por su parte, la CGC se asume como variable independiente, y se sustenta en la capacidad de infraestructura y la capacidad de proceso con que cuentan las instituciones educativas de nivel superior estudiadas.

En resumen, el conocimiento se reconoce como el recurso más importante para la ventaja competitiva y elemento clave para la innovación dentro de las organizaciones. La eficiencia productiva y los resultados operativos de la organización moderna dependen fundamentalmente de sus capacidades intelectuales. En consecuencia, surge un gran interés en cuanto a la implementación de la gestión del conocimiento como determinante de la capacidad de innovación y ventajas competitivas. 


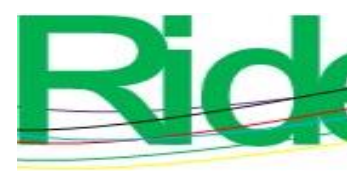

Revista Iberoamericana para la Investigación y el Desarrollo Educativo ISSN 2007-7467

Para las instituciones educativas de nivel superior, sean públicas o privadas, esta evidencia resulta relevante, de ahí sus esfuerzos para la implementación de estrategias dirigidas a gestionar conocimientos relevantes que permitan impulsar su capacidad de innovación y provean de ventajas competitivas en un mercado altamente globalizado y competitivo. Al igual que en las actividades productivas de bienes y servicios, en las organizaciones educativas el conocimiento se reconoce como el recurso más importante para la ventaja competitiva y la innovación, de ahí que estas organizaciones centren su atención en la gestión del conocimiento, activo intangible que mejora la capacidad de innovación.

La experiencia indica que la estrategia de gestión del conocimiento en el ámbito educativo tiene un impacto positivo en la innovación y el rendimiento a través de un aumento en la capacidad de innovación. Más allá de ello, en la educación existe una relación positiva y significativa entre la gestión del conocimiento y la innovación organizacional, así como entre la innovación organizacional y la ventaja competitiva sostenible.

\section{Material y métodos}

La presente investigación se sustentó en un enfoque cuantitativo de tipo descriptivo y confirmatorio; el propósito fue confirmar la existencia de una relación positiva entre la capacidad de gestión del conocimiento (CGC) y la capacidad de innovación (CIN) en las organizaciones educativas de nivel superior estudiadas. Para ello, se consideró a la CGC como variable independiente y a la CIN como variable dependiente. Una vez establecido el problema de investigación y fundamentadas teóricamente las variables CGC y CIN, se procedió a estudiar la relación existente entre ambos constructos. En el proceso se utilizaron herramientas de análisis estadístico descriptivo, factorial exploratorio y confirmatorio. En la modelación y prueba de hipótesis establecidas se aplicó un análisis de ecuaciones estructurales (SEM), el cual es más adecuado para este tipo de análisis (Herrero, 2010). El propósito principal fue explicar las covarianzas o correlaciones observadas entre un conjunto de variables medidas a través de un conjunto de variables latentes o factores (Bollen, 1989). 


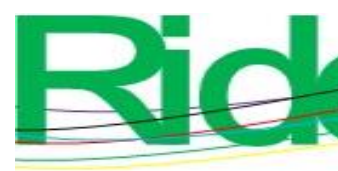

Revista Iberoamericana para la
Investigación y el Desarrollo Educativo
ISSN $2007-7467$

\section{Diseño}

El universo de estudio estuvo constituido por 77 instituciones de educación superior del sureste de México. En ellas laboran 8603 trabajadores administrativos, directivos y docentes. En la determinación del tamaño de muestra se aplicó muestreo probabilístico por conglomerados, con error de muestreo de $5 \%$, nivel de confianza de $95 \%$ y varianza de $\mathrm{p}=$ $.50, \mathrm{q}=.50$. Se obtuvo un tamaño de muestra de 441 empleados, de los cuales $73.7 \%$ laboraban en universidades públicas y el restante $26.3 \%$ en instituciones privadas. En el levantamiento de la información de campo se aplicó un cuestionario estructurado, diseñado a partir de los objetivos de la investigación. Con base en los hallazgos de la revisión bibliográfica se procedió a la operacionalización de las variables CGC y CIN. El instrumento quedó conformado por 88 ítems en escala de Likert. Para medir la CGC se utilizó la escala producida por Nguyen (2010), en tanto que en la medición de la CIN se utilizó la escala de Al-Husseini y Elbeltagi (2012). La confiabilidad de los ítems se evaluó mediante estimación de coeficiente alfa de Cronbach, según escala propuesta por Nunnelly (1978): menos de 0.6 (baja); entre 0.61 y 0.70 (adecuada); entre 0.71 a 0.80 (buena); mayor de 0.80 (alta). Para la validación del constructo se utilizaron el análisis factorial exploratorio (EFA) y el análisis factorial confirmatorio (CFA), según criterios de Hair, Gómez, Cano y Frances (2006). Finalmente, en el procesamiento y análisis de los datos se utilizó el programa SPSS (versión 21), así como análisis de ecuaciones estructurales en el estudio de relaciones causales entre los datos obtenidos, para lo cual se utilizó el paquete estadístico AMOSS (versión 20).

\section{Resultados}

\section{Descriptivos}

El análisis de la relación existente entre la CGC y la CIN se llevó a cabo en 77 instituciones de educación superior del sureste de México, de las cuales casi 74 \% son públicas y el restante $26 \%$ de capital privado (figura 1), y se aplicó a una muestra representativa de 441 trabajadores, de los cuales $55.1 \%$ eran hombres y $44.9 \%$ mujeres (figura 2). 
Figura 1. Datos descriptivos. Tipo de institución

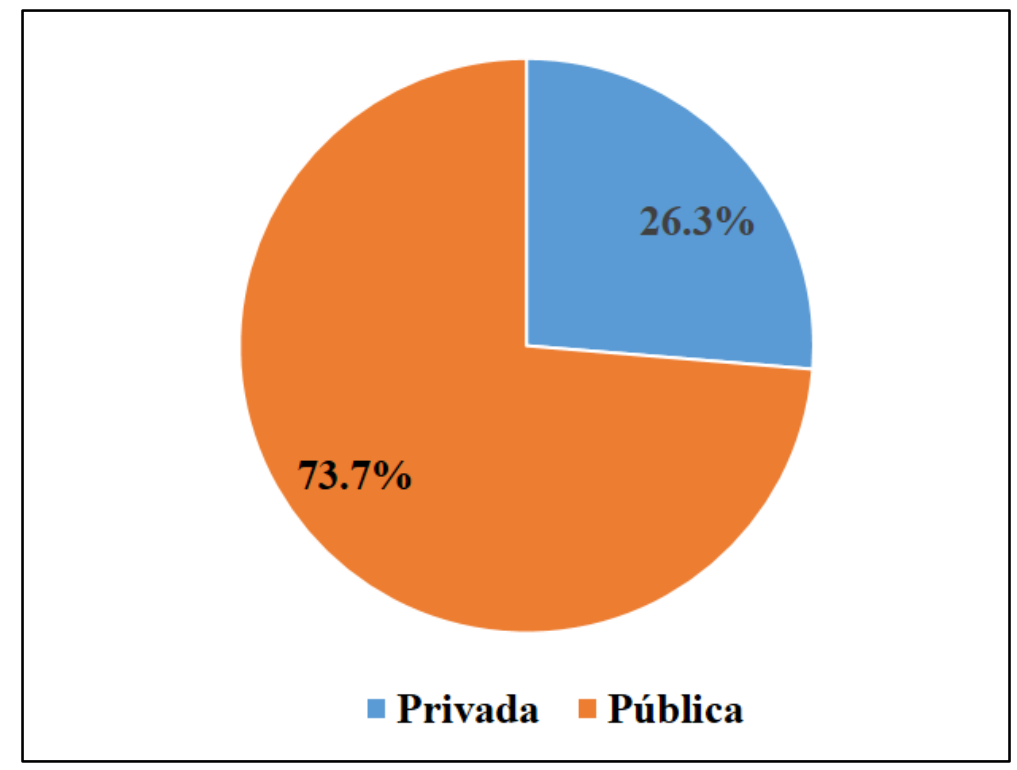

Fuente: Elaboración propia

Figura 2. Trabajadores según sexo

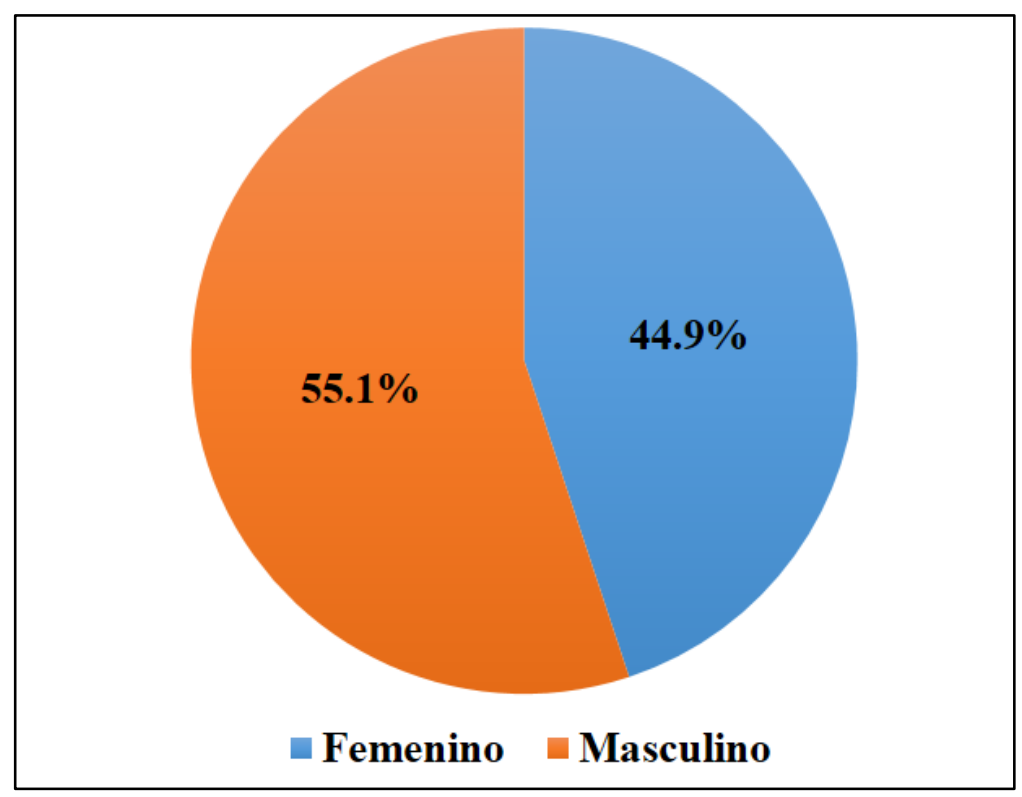

Fuente: Elaboración propia

El rango promedio de edad de los trabajadores estaba entre 41 y 45 años (figura 3), con un promedio de antigüedad laboral de 11 a 15 años (figura 4). 
Figura 3. Datos descriptivos (edad)

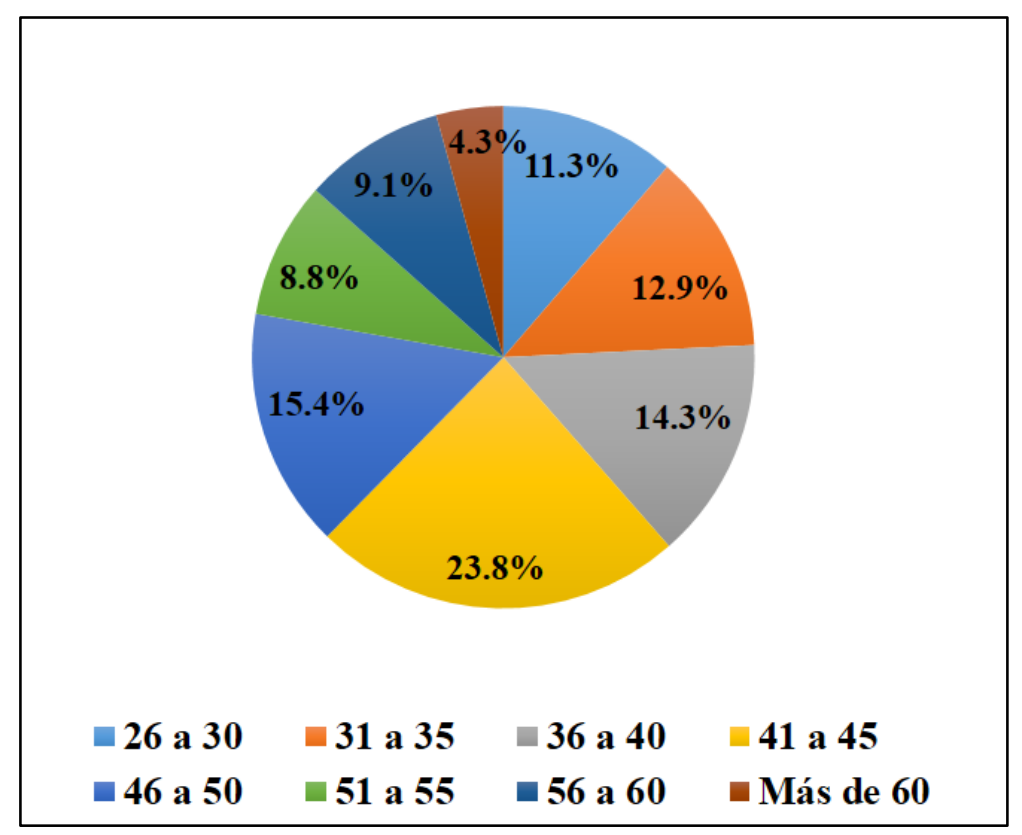

Fuente: Elaboración propia

Figura 4. Datos descriptivos (antigüedad)

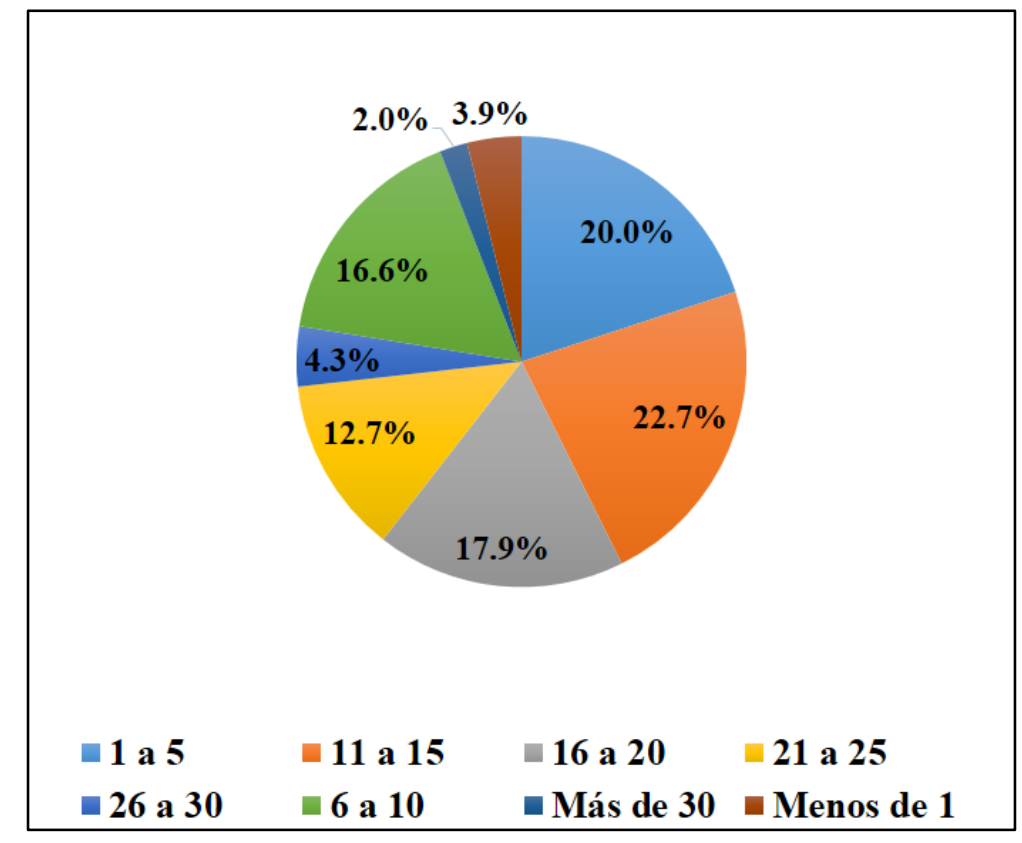

Fuente: Elaboración propia

El nivel de estudios promedio fue de maestría, con $50.5 \%$ del total. Al respecto, se destaca que $25.5 \%$ de los entrevistados contaba con doctorado, y $1.4 \%$ con diversas especialidades. Asimismo, $22.4 \%$ tenía estudios de licenciatura (figura 5). 


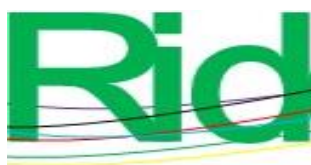

Revista Iberoamericana para la
nvestigación y el Desarrollo Educativo
ISSN $2007-7467$

Figura 5. Datos descriptivos (nivel de estudios)

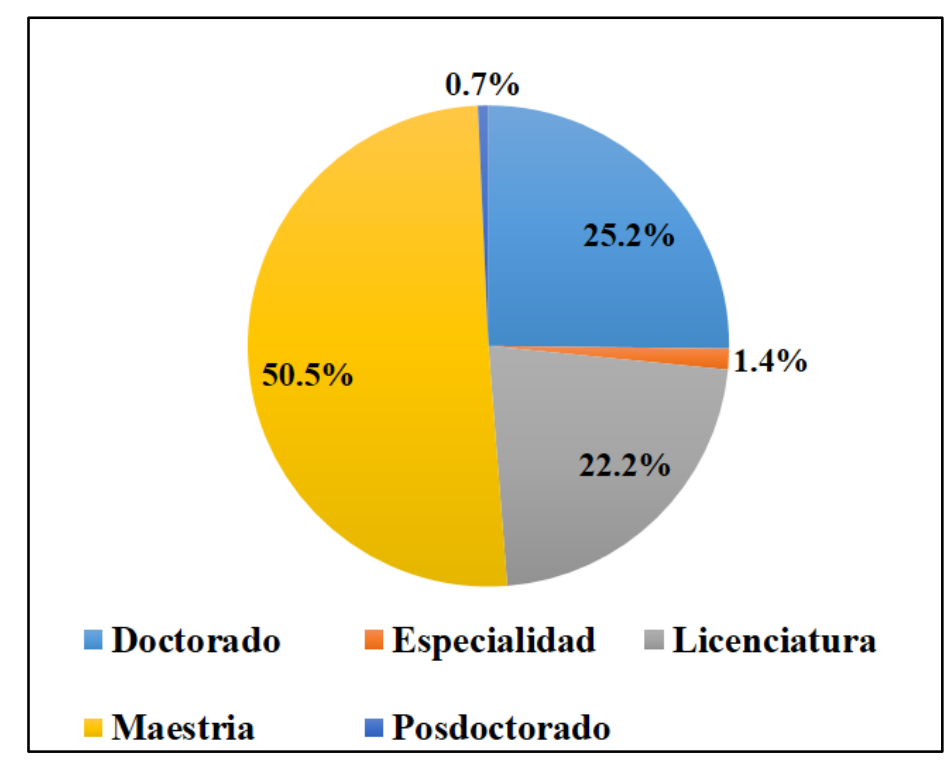

Fuente: Elaboración propia

Al momento de la entrevista, los trabajadores venían desempeñando principalmente la función docente (casi 57 \% ocupaba el cargo de profesor investigador tiempo completo y de asignatura). Se destacan también las actividades administrativas y administrativa docente con casi $37 \%$ del total. En menor orden de importancia se encuentran las funciones directivas, las cuales ocupaban el restante $6 \%$ (figura 6). 

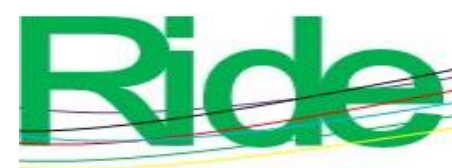

Revista Iberoamericana para la Investigación y el Desarrollo Educativo ISSN 2007 - 7467

Figura 6. Distribución de la muestra por cargo de las IES públicas y privadas

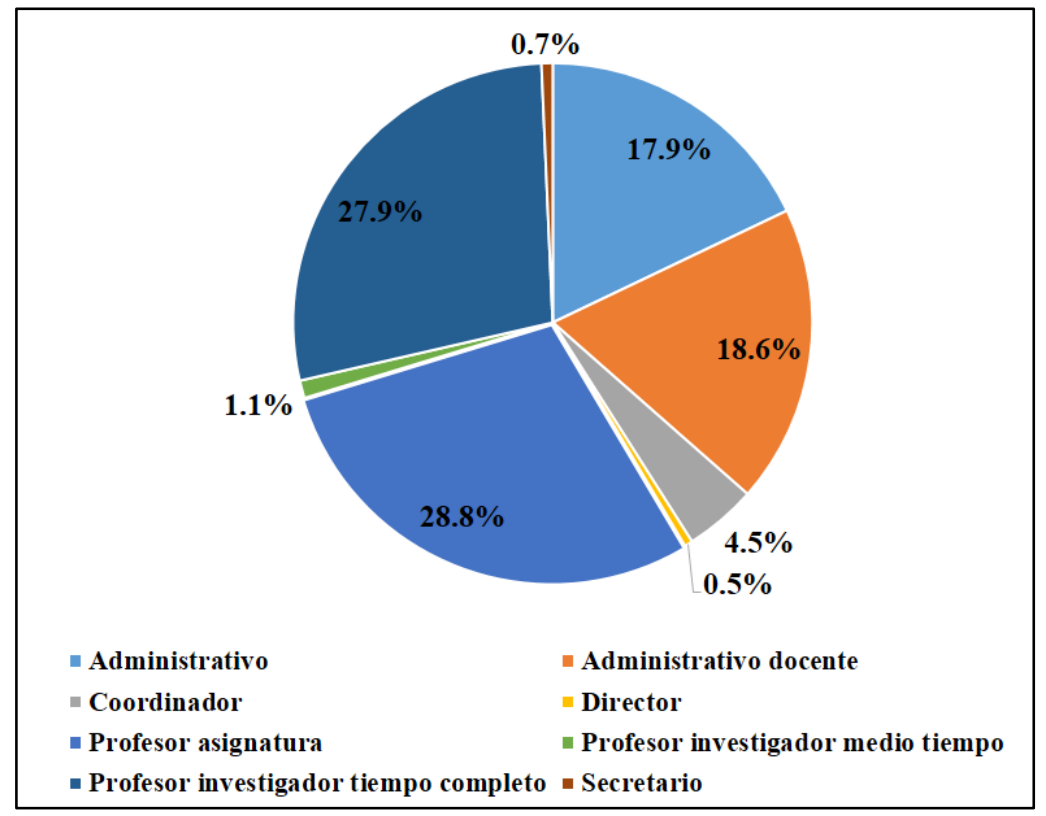

Fuente: Elaboración propia

Las instituciones educativas investigadas impartían preferentemente una amplia gama de estudios. En concreto, más de $51 \%$ ofrecían licenciaturas, diversas especialidades, maestrías y doctorados, y en poco más de $41 \%$ la oferta se centraba en licenciatura, especialidad y maestría (figura 7). 


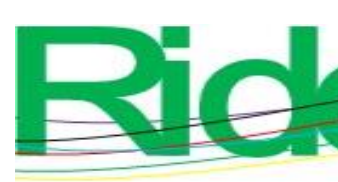

Revista Iberoamericana para la Investigación y el Desarrollo Educativo ISSN 2007 - 7467

Figura 7. Datos decriptivos (nivel educativo que imparte la institución)

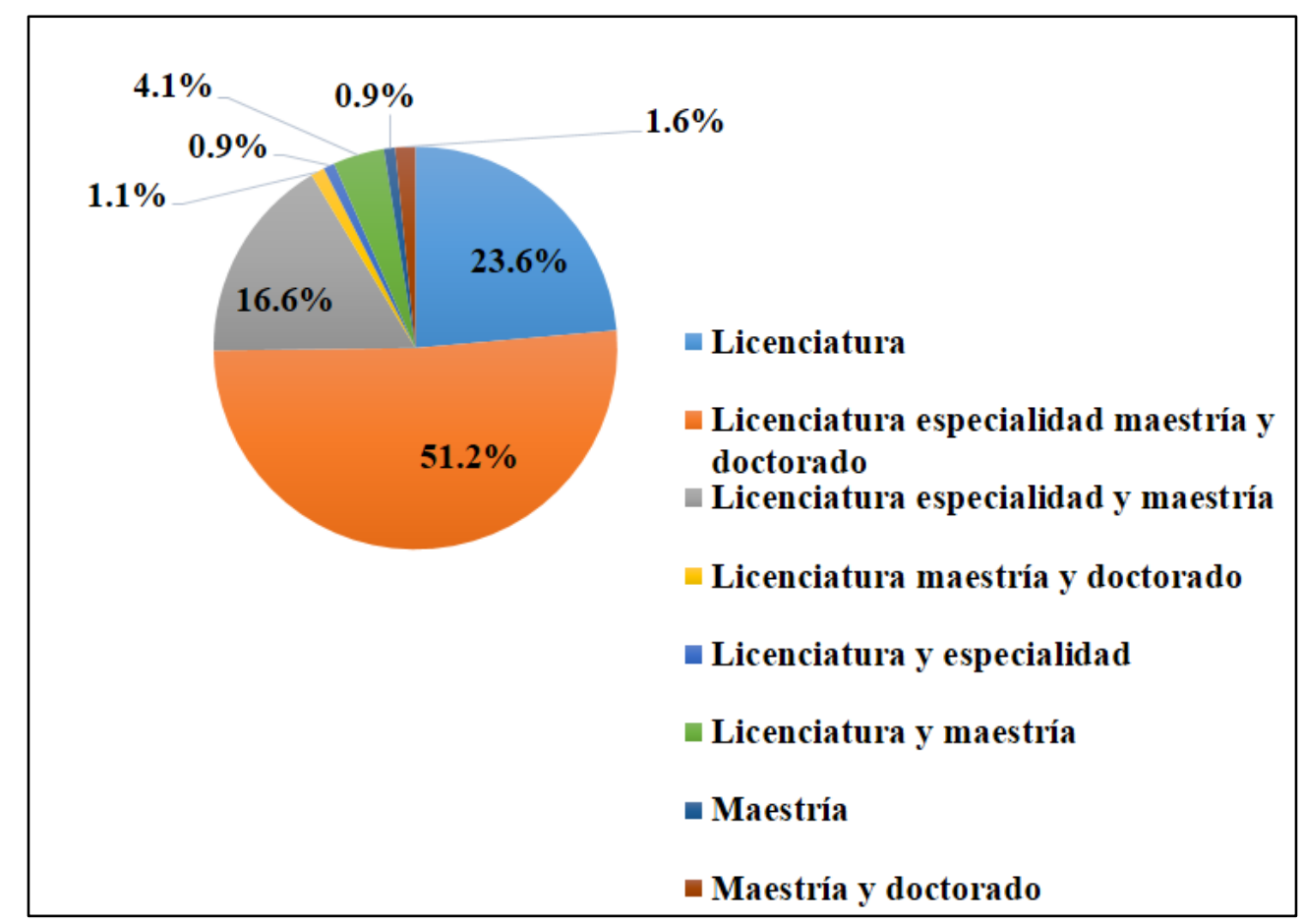

Fuente: Elaboración propia

\section{Confirmatorios}

Como se ha mencionado, el análisis desarrollado consideró a la CGC como variable independiente, la cual quedó compuesta por los constructos CEGC y CPGC, ya que se validó que ambos eran unidimensionales y estaban relacionados. Respecto a la CIN, fue determinada como variable dependiente, pues también se consideró su unidimensionalidad. Los resultados del SEM efectuado arrojaron un coeficiente de correlación de 0.921 entre ambas variables. Asimismo, la relación estimada (1.267), la estandarizada (.96) y el valor p (0.000), proporcionan evidencia de la existencia de una relación positiva entre ellas. Por lo tanto, se puede afirmar que la CGC influye positivamente en la CIN de las instituciones educativas de nivel superior estudiadas.

Estos resultados derivados de la evidencia empírica concuerdan con los alcanzados por Nonaka y Takeuchi (1995) en su investigación sobre la creación de conocimiento organizativo y su influencia en la capacidad de innovación en organizaciones educativas. Estos autores demuestran que el conocimiento constituye el requisito principal para la innovación y la ventaja competitiva de la institución. Específicamente, en estas 


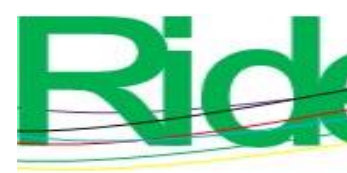

Revista Iberoamericana para la Investigación y el Desarrollo Educativo ISSN 2007 - 7467

organizaciones, la capacidad de gestión del conocimiento (CGC) a partir de la visión de los recursos y capacidades (VRC) y el enfoque basado en el conocimiento (EBC) constituye un recurso estratégico capaz de generar innovación y ventajas competitivas sostenibles (Grant, 1996). De esta manera, la introducción de programas de gestión del conocimietno en la organización educativa tiene un efecto positivo en la generación de innovación, ya que impulsa el desarrollo de habilidades institucionales, como son la adquisición de conocimiento y la conversión, difusión y aplicación interna del conocimiento acumulado (Palacios et al., 2009). En síntesis, en las organizaciones educativas de nivel superior, la gestión del conocimiento debe entenderse como un mecanismo institucional capaz de estimular la generación del conocimiento tácito y explícito que se difunde a través de la organización y su entorno, lo que se refleja directamente en su capacidad de innovación y ventaja competitiva.

En lo que corresponde a la relación existente entre la CEGC y la CPGC al interior de las instituciones educativas, se encontró evidencia suficiente para confirmar que la CEGC determina positivamente la CPGC. Esto es respaldado por la evidencia empírica, que muestra que las actividades generales de la organización englobadas en la CEGC son mecanismos que influyen determinantemente en la creación de conocimiento, así como en su protección e intercambio, conclusión coincidente con Leey Choi (2003) y Nguyen (2010). Asimismo, la dimensión de la CEGC que más influye en la CPGC es la cultura organizacional, la cual tiene una relación directa y significativa sobre la aplicación del conocimiento e indirecta con la adquisición y protección del conocimiento. De la misma manera, la estructura organizacional tiene también una relación indirecta sobre la conversión y aplicación del conocimiento.

\section{Discusión}

La revisión teórica efectuada, así como la evidencia empírica derivada de la investigación demuestran que la GC contribuye de manera determinante para que las organizaciones educativas apliquen de forma eficiente y efectiva el conocimiento y logren consolidar de mejor manera sus procesos de innovación. También se confirmó que existe una relación positiva y significativa entre la CGC y la CIN organizacional, y entre la innovación organizacional y la ventaja competitiva sostenible. Así, los resultados concuerdan con las conclusiones de Nonaka y Takeuchi (1995), quienes conciben el conocimiento como un recurso estratégico de la organización, así como para la innovación y ventaja competitiva 


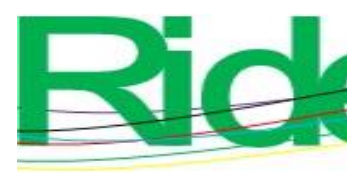

Revista Iberoamericana para la Investigación y el Desarrollo Educativo ISSN 2007 - 7467

sostenible (Grant, 1996). Asimismo, la puesta en marcha de programas de GC en la organización tiene un efecto positivo no solo sobre la innovación, sino que ayudan también a desarrollar habilidades para la adquisición de conocimientos, así como en su conversión, difusión y aplicación interna (Palacios et al., 2009). En este sentido, la GC debe entenderse como un mecanismo institucional capaz de estimular la coordinación de conocimiento tácito y explícito que se difunde a través de la organización y su entorno. Por lo tanto, la CGC está fuertemente vinculada con la CIN dentro de las organizaciones educativas (Palacios et al., 2009).

Los hallazgos de la investigación empírica sobre la influencia positiva y significativa de la CGC en la CIN en instituciones educativas coinciden con estudios preliminares de Noruzy, Dalfard, Azhdari, Nazari-Shirkouhi y Rezazadeh (2012) y Nouri, Mousavi y Soltan (2016). Los resultados demuestran también que la innovación requiere de actividades de creación de conocimiento a nivel organizacional (Shu et al., 2012); otros resultados evidencian que la aplicación del conocimiento tiene un efecto mediador en la innovación ( $\mathrm{Li}$, Liu, Wang, Li y Guo, 2009). La estrategia de GC tiene impacto positivo sobre la innovación y el rendimiento de la organización, a través de un aumento en la CIN (López y Meroño, 2011). La creación y adquisición de conocimiento es determinante en el rendimiento innovador (Zhang, Shu, Jiang y Malter, 2010), y la combinación del conocimiento impacta directamente la innovación de productos y de procesos (Shu et al., 2012).

En relación con el análisis de la CEGC, se observó que los resultados coinciden con Gold et al. (2001) y Lee y Choi (2003), quienes indican la existencia de una relación directa entre la cultura de conocimiento y la tecnología, e indirectamente con el recurso humano de la organización. Respecto a la estructura organizativa como fuente de estímulo a la GC dentro de las organizaciones educativas, el análisis efectuado indica la presencia de una relación indirecta con la adquisición, conversión y aplicación del conocimiento, resultado compatible con los hallazgos de Nonaka y Takeuchi (1995), Wang y Ahmed (2003) y Nguyen (2010). En cuanto a la tecnología, Gold et al. (2001) encuentran igualmente que esta representa un elemento trascendental de la dimensión estructural necesaria para la creación de nuevos conocimientos, ya que permite superar las barreras de comunicación presentes en la organización educativa. Asimismo, Gold et al. (2001) y Allameh, Zare y Davoodi (2011) encuentran que la tecnología de la información determina el acceso al conocimiento dentro 


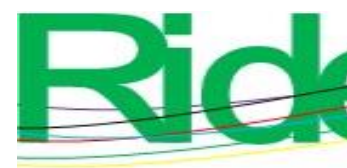

Revista Iberoamericana para la Investigación y el Desarrollo Educativo ISSN 2007 - 7467

de la organización educativa, y por lo tanto, esta debe invertir en la infraestructura tecnológica adecuada que respalde sus actividades científicas desarrolladas.

En cuanto al hallazgo de que en el interior de la estructura de las organizaciones educativas analizadas los recursos humanos facilitan el proceso de intercambio de conocimientos entre sus diversas áreas, este es compatible con Bharadwaj, Chauhan y Raman (2015), quienes encuentran que una estructura flexible permite la formación de equipos de trabajo colaborativo. Asimismo, Lee y Choi (2003) sostienen que el capital humano es la clave en la creación del conocimiento organizacional. Por ello, para Nguyen (2010) es fundamental el desarrollo continuo de las habilidades y competencias de los trabajadores de la organización.

En lo que respecta a cultura organizacional, el análisis demostró que se relaciona directamente con la aplicación del conocimiento e indirectamente con su adquisición y protección, lo que coincide con Gold et al. (2001), quienes encuentran que el mayor obstáculo para una eficaz GC es la ausencia de cultura organizacional. Asimismo, respecto a las tecnologías de la información (TI), el análisis estableció la existencia de una relación directa entre estas y la adquisición de conocimientos, hallazgo que coincide con los resultados de Lee y Choi (2003), quienes sostienen que las TI impactan positivamente en el conocimiento, facilitando la adquisición, almacenamiento e intercambio de conocimientos a gran escala, coadyuvando así al proceso de creación de conocimiento. Estos resultados son compatibles también con Hsu (2014) y Bharadwaj et al. (2015), quienes afirman que las TI constituyen un factor clave en la gestión del conocimiento.

En lo que corresponde a la CPGC, entendida como la capacidad de las organizaciones educativas para crear nuevos conocimientos, el análisis arroja la existencia de una relación directa y significativa, resultado compatible con el alcanzado por Nonaka y Takeuchi (1995). Por su parte, Lee y Suh (2003) encontraron que los procesos de gestión del conocimiento están orientados a lograr que el conocimiento existente sea útil para la organización.

Finalmente, respecto a la CIN en instituciones educativas, los resultados del estudio indican una relación directa y significativa entre la CGC y la CIN de productos y procesos educativos, datos que concuerdan con lo reportado por Shu et al. (2012) y Al-Husseini y Elbeltagi (2012), quienes demuestran que la capacidad de una organización para combinar y utilizar distintos tipos de conocimiento es esencial para lograr procesos efectivos de innovación. Asimismo, se encontró evidencia empírica de una relación directa entre la 


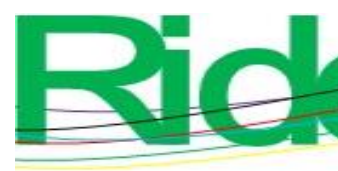

Revista Iberoamericana para la Investigación y el Desarrollo Educativo ISSN $2007-7467$

estructura organizacional y la innovación de procesos eductaivos, hallazgos compatibles con los indicados por Dilnutt (2000), quien encuentra que la estructura organizativa influye directamente en la gestión de conocimientos e innovación dentro de la organización. Al respecto, Nonaka y Takeuchi (1995) establecen que ciertos tipos de estructura facilitan el intercambio y generación de conocimiento, así como el impulso de la innovación.

\section{Conclusiones}

En un mundo más abierto, interconectado y competitivo, el conocimiento se ha convertido en factor clave para el desarrollo económico y social de todos los países. Por ello, el conocimiento, sobre todo el estructurado, es considerado como un recurso clave para las organizaciones, y su gestión y transferencia constituye un principio básico para su administración. En tal sentido, los resultados de la presente investigación demuestran que las instituciones de educación superior que gestionan los conocimientos utilizan de manera más eficiente los recursos con que cuentan, son más innovadoras y tienen un mejor desempeño. De la misma manera, la capacidad de innovación de estas organizaciones educativas está estrechamente relacionada con los activos intelectuales y conocimientos que posee. Asimismo, se evidencia una relación positiva y significativa entre su capacidad de generación de conocimiento y su capacidad de innovación, y esta capacidad de innovación impulsa su ventaja competitiva sostenible. Todas estas evidencias empíricas están ampliamente respaldadas en la revisión teórica efectuada.

En síntesis, la puesta en marcha de programas de generación de conocimiento en la organización educativas de nivel superior tiene un efecto positivo no solo sobre su capacidad de innovación, sino que contribuyen también a desarrollar habilidades para la adquisición de conocimientos, así como en su conversión, difusión y aplicación interna. En este sentido, la gestión del conocimiento debe entenderse como un mecanismo institucional capaz de estimular la generación del conocimiento tácito y explícito que se difunde a través de la organización y su entorno. Por lo tanto, la capacidad de generación de conocimiento está fuertemente vinculada con la capacidad de innovación dentro de las instituciones educativas de nivel superior. 


\section{Referencias}

Abdullah, S. and Hassan, W. (2013). The Impact of Human Resource Management Practices, Organisational Culture, Organisational Innovation and Knowledge Management on Organisational Performance in Large Saudi Organisations: Structural Equation Modeling With Conceptual Framework. International Journal of Business and Management, 8(22), 1-19. Doi: 10.5539/ijbm.v8n22p1

Al-Husseini, S. and Elbeltagi, I. (2012). Knowledge Sharing and Innovation: An Empirical Study in Iraqi Private Higher Education Institutions. International Conference on Intellectual Capital. Knowledge Management \& Organizational Learning. 129-138.

Allameh, S., Zare, S. and Davoodi, S. (2011). Examining the Impact of KM Enablers on Knowledge. Management Processes, Procedia Computer Science, 3, 1211-1223. Doi: 10.1016/j.procs.2010.12.196

Barragán, A. (2009). Aproximación a una taxonomía de modelos de gestión del conocimiento. Intangible Capital, 5(1), 65-101. Doi: 10.3926/ic.2009.v5n1.p65-101

Bharadwaj, S., Chauhan, S. and Raman, A. (2015). Impact of Knowledge Management Capabilities on Knowledge Management Effectiveness in Indian Organizations. VIKALPA, The Journal for Decision Makers, 40(4), 421-434. Doi: $10.1177 / 0256090915613572$

Bidmeshgipour M., Khairuzzaman W. and Omar, R. (2012). Knowledge management and organizational innovativeness in Iranian banking industry. Knowledge Management \& E-Learning: An International Journal, 4(4), 481-499.

Bollen, K. A. (1989). Structural equations with latent variables. New York: John Wiley y Sons.

Bornay, M., De la Rosa, D., López, A. and Valle, R. (2012). Employment Relationships and Firm Innovation: The Double Role of Human Capital. British Journal of Management, 23, 223-240. Doi: 10.1111/j.1467-8551.2010.00735.x

Cabello, C., López, A. and Valle, R. (2011). Leveraging the innovative performance of human capital through HRM and social capital in Spanish firms. The International Journal of Human Resource Management, 22(4), 807-828. Doi: $10.1080 / 09585192.2011 .555125$ 


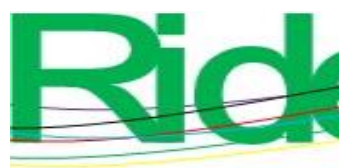

Revista Iberoamericana para la Investigación y el Desarrollo Educativo ISSN $2007-7467$

Camelo, C., García, J., Sousa, E. and Valle, R. (2011). The influence of human resource management on knowledge sharing and innovation in Spain: the mediating role of affective commitment. The International Journal of Human Resource Management, 22(7), 1442-1463. Doi: 10.1080/09585192.2011.561960

Chuang, S. (2004). A resource-based perspective on knowledge management capability and competitive advantage: an empirical investigation. Expert Systems with Applications, 27(3), 459-465. Doi: 10.1016/j.eswa.2004.05.008

Damanpour, F., Walker, R. and Avellaneda, C. (2009). Combinative Effects of Innovation Types and Organizational Performance: A Longitudinal Study of Service Organizations. Journal of Management Studies, 46(4), 650-675. Doi: 10.1111/j.14676486.2008.00814.x

Darroch, J. (2005). Knowledge management, innovation and firm performance. Journal of knowledge Management, 9(3), 101-115. Doi: 10.1108/13673270510602809

Davenport, T. and Prusak, L. (1998). Working Knowledge: How Organizations Manage What They Know. Boston, MA: Harvard Business School Press.

De Long, D. and Fahey, L. (2000). Diagnosing cultural barriers to knowledge management. Academy of Management Executive, 14(4), 113-127. Doi: 10.5465/AME.2000.3979820

Dilnutt, R. P. (2000). Knowledge management as practiced in Australian organizations: a case study approach (DBA thesis). Australia: Southern Cross University.

Drucker, P. F. (1993). Innovation and Entrepreneurship (Harper Business edition, first edition 1986). New York; HarperCollins Publishers.

El-Kot, G. y Gamal, D. (2011). How Does Knowledge Management Drive Competitiveness in Egyptian Software Companies? Review of Management, 1(4), 56-76.

Gold, A., Malhotra, A. and Segars, A. (2001). Knowledge management: An organizational capabilities perspective. Journal of Management Information Systems, 18(1), 185214. Doi: 10.1080/07421222.2001.11045669

Grant, R. (1996). Toward a Knowledge-Based Theory of the Firm. Strategic Management Journal, 17, 109-122. Doi: 10.1002/smj.4250171110

Hair, J. F., Gómez, S, M., Cano, D. and Frances, P. E. (2006). Analisis multivariante. Madrid: Prentice Hall. 


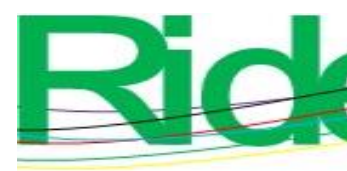
Revista Iberoamericana para la
Investigación y el Desarrollo Educativo
ISSN $2007-7467$

Haiyang, L. and Kwaku, A. (2001). Product innovation strategy and the performance of new technology ventures in China. Academy of Management Journal, 44(6), 1123-1134. Doi: $10.2307 / 3069392$

Herrero, J. (2010). El análisis factorial confirmatorio en el estudio de la estructura y estabilidad de los instrumentos de evaluación: un ejemplo con el cuestionario de autoestima CA-14. Psychosocial Intervention, 19(3), 289-300.

Hsu, S. (2014). Effects of Organization Culture, Organizational Learning and IT Strategy on Knowledge Management and Performance. The Journal of International Management Studies, 9(1), 50-58.

Lee, H. and Suh, Y. (2003). Knowledge conversion with information technology of Korean companies. Business Process Management Journal, 9(3), 317-336. Doi: $10.1108 / 14637150310477911$

Lee, H. and Choi, B. (2003). Knowledge management enablers, processes, and organizational performance: an integrative view and empirical examination. Journal of Management Information. Systems, 20(1), 179-228. Doi: 10.1080/07421222.2003.11045756

Lee, V., Leong, L., Hew, T. and Ooik, K. (2013). Knowledge management: a key determinant in advancing technological innovation? Journal of Knowledge Management, 17(6), 848-872. Doi: 10.1108/JKM-08-2013-0315

Li, Y., Liu, X., Wang, L., Li, M. and Guo, H. (2009). How Entrepreneurial Orientation Moderates the Effects of Knowledge Management on Innovation. Systems Research and Behavioral Science, 26, 645-660. Doi: 10.1002/sres.980

López, C. and Meroño A. L. (2011). Strategic knowledge management innovation and performance. International Journal of Information Management, 31(6), 502-509. Doi: 10.1016/j.ijinfomgt.2011.02.003

Mehrabani, S. E. and Shajari, M. (2012). Knowledge Management and Innovation Capacity. Journal of Management Research, 4(2), 164-177. Doi: 10.5296/jmr.v4i2.1390

Nguyen, T. (2010). Knowledge management capability and competitive advantage: an empirical study of Vietnamese enterprises (PhD thesis). Southern Cross University, Lismore, NSW.

Nonaka, I. and Takeuchi, H. (1995). The knowledge-creating company: How Japanese companies create the dynamics of innovation. Oxford, London: Oxford University Press. 


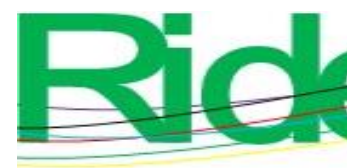

Revista Iberoamericana para la Investigación y el Desarrollo Educativo ISSN $2007-7467$

Noruzy, A., Dalfard, V. M., Azhdari, B., Nazari-Shirkouhi, S. and Rezazadeh, A. (2012). Relations between transformational leadership organizational learning knowledge management organizational innovation, and organizational performance: an empirical investigation of manufacturing firms. International Journal of Advanced Manufacturing Technology, 64, 1073-1085. Doi: 10.1007/s00170-012-4038-y

Nouri, B. A., Mousavi, M. M. and Soltan, M. (2016). Effect of Transformational Leadership and Knowledge Management Processes on Organizational Innovation in Ardabil University of Medical Sciences. International Journal of Management, Accounting and Economics, 3(11), 672-698.

Nunnelly, J. C. (1978). Psycometric Theory (2 ${ }^{\text {th }}$ ed.). New York: McGraw Hill.

Palacios, D., Gil, I. and Garrigos, F. (2009). The impact of knowledge management on innovation and entrepreneurship in the biotechnology and telecommunications industries. Small Business Economics, 32, 291-301. Doi: 10.1007/s11187-008-91466

Piri, M., Jasemi, M. and Abdi, M. (2013). Intellectual capital and knowledge management in the Iranian space industries. VINE: The Journal of Information and Knowledge Management Systems, 43(3), 341-356. Doi: 10.1108/VINE-08-2012-0036

Prajogo, D. and Pervaiz, A. (2006). Relationships between innovation stimulus, innovation capacity, and innovation performance. $R \& D$ Management, 36(5), 449-515. Doi: 10.1111/j.1467-9310.2006.00450.x

Reza, M. and Pahlavani, N. (2013). The effect of social capital on knowledge creation in Petrochemical Industry. Management Science Letters, 3, 879-884. Doi: 10.5267/j.msl.2013.01.034

Salete, J., Tcholakian, A. B. y Selig, P. M. (2013). A influência do capital intelectual no potencial de inovação das universidades. Iberoamerican Journal of Industrial Engineering, 5(9), 01-22.

Segarra, M. (2006). Estudio de la naturaleza estratégica del conocimiento y las capacidades de gestión: aplicación a empresas innovadoras de base tecnología (tesis doctoral). Universitat Jaumet, Castellon, España.

Shu, Ch., Page, A., Gao, S. y Jiang, X. (2012). Managerial Ties and Firm Innovation: Is Knowledge Creation a Missing Link?. Product Development \& Management Association, 29(1), 125-143. Doi: 10.1111/j.1540-5885.2011.00883.x 

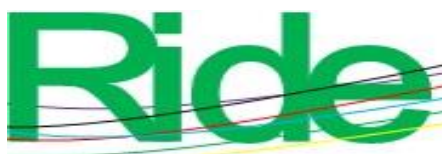

Revista Iberoamericana para la Investigación y el Desarrollo Educativo

ISSN $2007-7467$

Swan, J. and Newell, S. (2000). Linking Knowledge Management and innovation. European Conference on Information Systems, 173, 591-598.

Wang, C. and Ahmed, P. (2003). Structure and structural dimensions for knowledge-based organization's. Measuring Business Excellence, 7(1), 51-62. Doi: $10.1108 / 13683040310466726$

Wang, C. and Ahmed, P. (2004). The development and validation of the organisational innovativeness construct using confirmatory factor analysis. European Journal of Innovation Management, 7(4), 303-313. Doi: 10.1108/14601060410565056

Zhang, H., Shu, Ch., Jiang, X. and Malter, A. J. (2010). Managing Knowledge for: The Role of Cooperation, Competition, and Alliance Nationality. Journal of International Marketing, 18(4), 74-94. Doi:10.1509/jimk.18.4.74 


\begin{tabular}{|c|c|}
\hline Rol de Contribución & Autor (es) \\
\hline Conceptualización & JOSÉ FÉLIX GARCÍA RODRÍGUEZ \\
\hline Metodología & GEORGINA GUADALUPE SHRINER SIERRA \\
\hline Software & NO APLICA \\
\hline Validación & $\begin{array}{l}\text { DAVID MARTÍNEZ LUIS (IGUAL) } \\
\text { IGNACIO CAAMAL CAUICH (IGUAL) }\end{array}$ \\
\hline Análisis Formal & $\begin{array}{l}\text { JOSÉ FÉLIX GARCÍA RODRÍGUEZ (PRINCIPAL) } \\
\text { GEORGINA GUADALUPE SHRINER SIERRA (APOYO) }\end{array}$ \\
\hline Investigación & $\begin{array}{l}\text { JOSÉ FÉLIX GARCÍA RODRÍGUEZ (PRINCIPAL) } \\
\text { GEORGINA GUADALUPE SHRINER SIERRA (APOYO) }\end{array}$ \\
\hline Recursos & NO APLICA \\
\hline Curación de datos & NO APLICA \\
\hline $\begin{array}{l}\text { Escritura - Preparación del } \\
\text { borrador original }\end{array}$ & JOSÉ FÉLIX GARCÍA RODRÍGUEZ \\
\hline $\begin{array}{l}\text { Escritura - Revisión y } \\
\text { edición }\end{array}$ & $\begin{array}{l}\text { JOSÉ FÉLIX GARCÍA RODRÍGUEZ (PRINCIPAL) } \\
\text { GEORGINA GUADALUPE SHRINER SIERRA (APOYA) }\end{array}$ \\
\hline Visualización & NO APLICA \\
\hline Supervisión & GEORGINA GUADALUPE SHRINER SIERRA \\
\hline $\begin{array}{l}\text { Administración de } \\
\text { Proyectos }\end{array}$ & JOSÉ FÉLIX GARCÍA RODRÍGUEZ \\
\hline Adquisición de fondos & IGNACIO CAAMAL CAUICH \\
\hline
\end{tabular}

